\title{
MicroRNA-338 inhibits migration and proliferation by targeting hypoxia-induced factor $1 \alpha$ in nasopharyngeal carcinoma
}

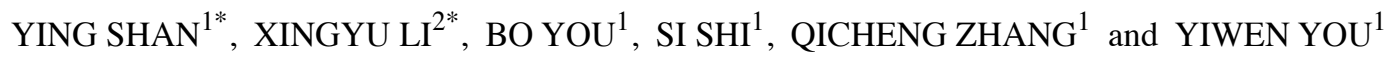 \\ ${ }^{1}$ Department of Ear, Nose and Throat, Affiliated Hospital of Nantong University, Nantong, Jiangsu 226001; \\ ${ }^{2}$ Department of Pathology, Medical School of Nantong University, Nantong, Jiangsu 226001, P.R. China
}

Received June 18, 2015; Accepted July 27, 2015

DOI: $10.3892 /$ or.2015.4195

\begin{abstract}
Nasopharyngeal cancer (NPC) is an endemic type of head and neck cancer with a high rate of cervical lymph node metastasis. An increasing number of studies have shown that microRNAs (miRNAs) play a key role in the development and progression of NPC. miR-338-3p has been demonstrated as an anti-oncogene in different solid tumors. The aim of the present study was to investigate the potential role of miR-338-3p in the development and progression of NPC. Compared with normal samples, our data showed that miR-338-3p were downregulated in NPC tissues and cells. The luciferase assay demonstrated that HIF- $1 \alpha$ was a direct target of miR-338-3p. We also found that miR-338-3p regulated the expression levels of HIF-1 $\alpha$, respectively. Overexpression of miR-338-3p in NPC cells significantly inhibited cell proliferation, and migration. Conversely, miR-338-3p knockdown in cells with lower endogenous expression levels significantly reduced antitumor behavior. Furthermore, enforced expression of miR-338-3p led to a decline in ERK phosphorylation as well as inhibited the hypoxia induced epithelial to mesenchymal transition. Cells pre-transfected with miR-338-3p can overcome hypoxiamediated cisplatin resistance. Taken together, we found that miR-338-3p directly targeted HIF-1 $\alpha$, and we provide insight into NPC initiation and progression, possibly representing a novel therapeutic target.
\end{abstract}

\section{Introduction}

Nasopharyngeal carcinoma (NPC) is a common head and neck cancer derived from epithelium cells which were located

Correspondence to: Dr Yiwen You, Department of Ear, Nose and Throat, Affiliated Hospital of Nantong University, 20 Xisi Road, Nantong, Jiangsu 226001, P.R. China

E-mail: youyiwen_nantong@163.com

${ }^{*}$ Contributed equally

Key words: miR-338-3p, metastasis, nasopharyngeal cancer, HIF-1 $\alpha$, proliferation in the nasopharynx (1). NPC is notorious for its metastatic potential at the early stages of the disease via both lymph and blood vessels. Due to the recurrence and distant metastasis, the prognosis of NPC patients is very poor (1). Furthermore, drug resistance may hamper the efficacy of anti-cancer drugs (2). Therefore, there is a great and urgent need to develop early diagnostic or predictive markers for NPC and to elucidate the mechanisms that would allow the development of efficient treatment options.

Recent studies of microRNAs (miRNAs) suggest a potential role of these regulatory molecules as candidate oncogenes or tumor suppressors in cancer progression (3-5). miRNAs are small non-coding RNA molecules $\sim 19-25$ nucleotide, which exist in many organisms and regulate gene expression through degradation of the corresponding mRNAs and/or inhibiting their translation by binding to their 3'-UTRs $(1,6,7)$. miRNAs have been reported to participate in a variety of pathways in physiological and pathological processes such as cellular differentiation, proliferation and apoptosis $(1,8)$, metastasis and resistance to therapy (6). Dysregulated expression of miRNAs has been reported in most tumor types, including NPC (1). At present, several miRNAs have been shown to target specific mRNAs to regulate the progression of NPC (9). For example, miR-29c suppressed metastasis by targeting TIAM1 and enhanced the sensitivity to cisplatin based chemotherapy and radiotherapy in nasopharyngeal carcinoma $(10,11)$. miR-451, by targeting MIF, inhibited cell growth and invasion and was associated with survival of NPC (1). Furthermore, miR-218 (12), miR-26a/b (13,14), miR-216b (15), miR-10b (16), miR-141 (17) and miR-200a (18) have been shown to have tumor-suppressive functions in NPC. The dysregulated miRNAs are involved in NPC development and progression by regulating cell growth, proliferation, apoptosis, invasion and metastasis (1), indicating that miRNAs play important roles in NPC tumorigenesis.

Recently, there has been increasing interest in miR-338-3p as an anti-oncogene in different solid tumors, including gastric cancer (19), hepatocellular carcinoma (20) and malignant melanoma (21). For example, miR-338-3p suppressed liver cancer cell invasion by targeting smoothened (7). Moreover, miR-338 can decrease proliferative and migratory, invasive behavior by attenuating the expression of NRP1 in gastric cancer (22). However, there is no study on the functions and mechanisms of miR-338-3p in NPC development and progression. 
Hypoxia or low oxygen tension has emerged as a specific and general feature of the microenvironment of many malignant tumors (23), including nasopharyngeal cancer (24). Tumor hypoxia is known to be mainly responsible for tumor resistance to radiotherapy and chemotherapy as well as to promote tumor phenotype influencing invasiveness, metastasis and poor prognosis (24). Studies showed that $100 \%$ of primary NPC and $58 \%$ of cervical nodal metastases of NPC were found with hypoxic regions which increased distant metastases, as well as resistance to chemotherapy in advanced NPC patients (25). There is emerging evidence shows that the adaptive response to hypoxia is also mediated by HIF-1dependent pathways in cancer as well as in nasopharyngeal carcinoma $(24,26)$. Among the proteins associated with tumor hypoxia, hypoxia-inducible factor 1 -alpha (HIF-1 $\alpha$ ) is an important hypoxia regulatory molecule promoting angiogenesis and metastasis (25). HIF-1 $\alpha$ is commonly found overexpressed in NPC (27). HIF-1 $\alpha$ has been reported as a prognostic factor as well as a potential therapeutic target of NPC (25). HIF-1 $\alpha$ can be transcriptionally and translationally regulated by signaling molecules such as cytokines growth factors and microRNAs (28). However, the association of HIF-1 $\alpha$ with miR-338-3p has not been established in NPC.

In the present study, we found that decreased expression of miR-338-3p had a causal role as a tumor suppressor in NPC by target HIF-1 $\alpha$. miR-338-3p was downregulated in NPC tissues and cells. We found that miR-338-3p regulated the expression levels of HIF-1 $\alpha$ of CNE2 cells under hypoxia, respectively. The luciferase assay showed that HIF-1 $\alpha$ was a direct target of miR-338-3p. Overexpression of miR-338-3p suppressed the proliferation and migration of CNE2 cells under hypoxia, whereas the inhibition of miR-338-3p promoted these processes. Furthermore, our results suggested that the chemosensitizing effect of miR-338-3p may be an important feature for its potential therapeutic roles in NPC.

\section{Materials and methods}

Clinical specimens and cell culture. All NPC specimens and normal nasopharyngeal epithelium samples were obtained from the Affiliated Hospital of Nantong University. No patients had received any antitumor treatments before biopsy. The patients were diagnosed via histopathological evidence. The research protocols were approved by the Academic Committee of Nantong University.

The human immortalized nasopharyngeal epithelial cell line NP69 and NPC cell line CNE2 were received as a kind gift from the Sun Yat-Sen University of China. The NPC cell lines CNE1 and 5-8F, 6-10B were a kind gift from the Xiang-Ya School of Medicine, Central South University, China. The human immortalized nasopharyngeal epithelial cell line NP69 was maintained in keratinocyte/serum-free medium (Invitrogen) supplemented with bovine pituitary extract (BD Biosciences), human NPC cell lines (CNE-1, CNE-2, 5-8F and 6-10B) were cultured in RPMI-1640 (Gibco) supplemented with 10\% FBS (Gibco). 5-8F and 6-10B cells were cultured in completed medium with $100 \mathrm{U} / \mathrm{ml}$ penicillin and $100 \mu \mathrm{g} /$ $\mathrm{ml}$ streptomycin (Shanghai Genebase Gen-Tech Co., Ltd., Shanghai, China). All the cells were cultured at $37^{\circ} \mathrm{C}$ in a $5 \%$ $\mathrm{CO}_{2}$ incubator.
Hypoxic condition was induced by exposing the cells in a hypoxia modular incubator chamber (Billups-Rothenberg) that provided hypoxic conditions with $1 \% \mathrm{O}_{2}, 5 \% \mathrm{CO}_{2}$ and $94 \% \mathrm{~N}_{2}$.

Cell transfection. CNE2 cells were seeded in 6-well plates and cultured overnight at $37^{\circ} \mathrm{C}$ in a humidified atmosphere of $95 \%$ air and $5 \% \mathrm{CO}_{2}$. Subsequently, transfections were conducted for miR-338-3p mimics, miR-338-3p inhibitor and non-specific control (NC) using HiPerFect transfection reagent (Qiagen) according to the manufacturer's instructions. Following culture for a further $48 \mathrm{~h}$, total RNA and cellular protein lysates were collected and used for reverse transcription-quantitative PCR (RT-qPCR) and western blot analysis, respectively.

Quantitative real-time PCR ( $q R T-P C R)$ analysis. miR-338-3p expression in NPC cells and tissues compared with normal was measured with SYBR qRT-PCR. Total RNA was extracted with TRIzol (Invitrogen) according to the manufacturer's instructions. miR-388-3p cDNA was synthesized from $2 \mu \mathrm{g}$ of total RNA. Subsequently, mRNA expression was analyzed by quantitative PCR using primers designed and synthesized by Guangzhou RiboBio Co., Ltd. (Guangzhou, China). Briefly, $20 \mu 1$ reactions containing $2 \mu 1$ RT product, $9 \mu 1$ of SYBRGreen PCR Master Mix and $200 \mathrm{nM}$ of primers were subjected to $1 \mathrm{cycle}$ of $95^{\circ} \mathrm{C}$ for $20 \mathrm{sec}$, and then 40 cycles of $95^{\circ} \mathrm{C}$ for $10 \mathrm{sec}, 60^{\circ} \mathrm{C}$ for $20 \mathrm{sec}$ and $70^{\circ} \mathrm{C}$ for $10 \mathrm{sec}$. miR-338-3p expression was normalized to U6 RNA. The $2^{-\Delta \Delta C T}$ method was used to quantify the expression changes of target genes. Three independent experiments were performed.

Western blot analysis. HIF-1 $\alpha$ proteins were measured by western blot analysis. Total protein was extracted from transfected cells using the RIPA lysis buffer (Beyotime Institute of Biotechnology, Haimen, China) according to the manufacturer's instructions. For western blot analysis, equal amounts of protein samples $(20 \mu \mathrm{g})$ were separated by $10 \%$ SDS-PAGE and transferred onto PVDF membranes (Millipore, Billerica, MA, USA). Membranes were blocked using with 5\% skim milk in TBST. After $2 \mathrm{~h}$ at room temperature, the membranes were incubated overnight with polyclonal primary antibodies. The antibodies used were as follows: anti-HIF-1 $\alpha$ (Abcam; 1:2,000 dilution), anti-p-ERK (Santa Cruz Biotechnology; 1:500 dilution), anti-ERK (Santa Cruz Biotechnology; 1:500 dilution) and mouse anti- $\beta$-actin (Santa Cruz Biotechnology; 1:1,000 dilution). Blots were then incubated with goat anti-rabbit or anti-mouse secondary antibody conjugated to horseradish peroxidase (Santa Cruz Biotechnology; 1:1,000 dilution) and visualized by enhanced chemiluminescence (ECL; Cell Signaling Technology). The values are representative of at least three independent experiments.

Luciferase reporter assay. HEK293 cells were seeded in the inner wells of 24-well plates. Then, cells were co-transfected using Lipofectamine 2000 (Invitrogen) with $80 \mathrm{ng}$ of the pGL3vector plasmid harboring the wild-type or mutant $3^{\prime}$-UTR of HIF-1 $\alpha$ and $4 \mathrm{ng}$ of the pRL-TK. For each plate, the hsamiR-338-3p mimics or mimics NC (Biomics Biotechnologies Co., Ltd., Nantong, China) was co-transfected at a final concentration of 50 pmol. Luciferase activities were measured 
consecutively $48 \mathrm{~h}$ post-transfection using the Dual-Luciferase reporter assay system (Promega, Southampton, UK) according to the manufacturer's instructions. Luminescence signals served as a measure for reporter activity normalized for transfection efficiency.

Wound healing assay. Wound healing assay was conducted $48 \mathrm{~h}$ after cell transfection. An artificial homogeneous wound was created on the monolayer using a sterized $200 \mu 1$ micropipette tip when the cells reached $\sim 90 \%$ confluency. Cell debris was removed by washing with RPMI-1640 twice. Wound closure was recorded and observed by light microscopy and images were captured at the indicated time-points.

Immunocytochemical analysis. Cells cultured on glass coverslips were transfected with miR-338-3p mimics, miR-338-3p inhibitor and non-specific control (NC) and then cultured under hypoxia condition for $24 \mathrm{~h}$. Glass coverslips were fixed with $4 \%$ paraformaldehyde and washed in PBS. The cells were incubated with rabbit anti-HIF-1 $\alpha$ antibody (Abcam; 1:200 dilution) overnight at $4^{\circ} \mathrm{C}$. PBS washed and incubated with fluorescein isothiocyanate (FITC) labeled secondary antibodies (EarthBox, Lancaster, PA, USA; 1:1,000) and the same time the nuclei were labeled with Hoechst (Invitrogen, Carlsbad, CA, USA). The coverslips were then observed under an Olympus camera.

Cell viability assay (Cell Counting kit-8). Cells were seeded into 96-well plates $\left(1 \times 10^{4}\right.$ cells/well) and treated as indicated. Cell viability was assessed by Cell Counting kit- 8 assay (Beyotime Institute of Biotechnology, Shanghai, China). The absorbance of each well was read on a microplate reader (F-2500 fluorescence spectrophotometer; Hitachi) at $450 \mathrm{~nm}$. Three independent experiments were performed in quintuplicate.

Migration assays. For the Transwell migration assays, 5x10 CNE2 cells were plated in a serum-free medium in the top chamber with a non-coated membrane (24-well insert; $8 \mu \mathrm{m}$ pore size; Millipore) and a medium supplemented with $10 \%$ serum was in the lower chamber. The cells were incubated for $24 \mathrm{~h}$ under hypoxia conditions. The non-migrated cells were removed from the upper sides of the Transwell membrane filter inserts using cotton-tipped swabs. The migrated/invaded cells on the lower sides were stained with crystal violet and the cells were counted.

Flow cytometry. Cells were plated in 6-well plates at a specific density. The cells were treated with miR-338-3p mimics, inhibitor or miR-NC under hypoxic conditions for $24 \mathrm{~h}$. After $24 \mathrm{~h}$, the cells were collected and analyzed using an Annexin V-FITC apoptosis detection kit (BD Biosciences, Oxford, UK). The apoptotic cells were detected by flow cytometry.

Statistical analysis. All the data are expressed as mean \pm SEM. Data were compared using the Student's t-test. $\mathrm{P}<0.05$ was considered to indicate a statistically significant difference. Each experiment consisted of at least three replicates per condition.

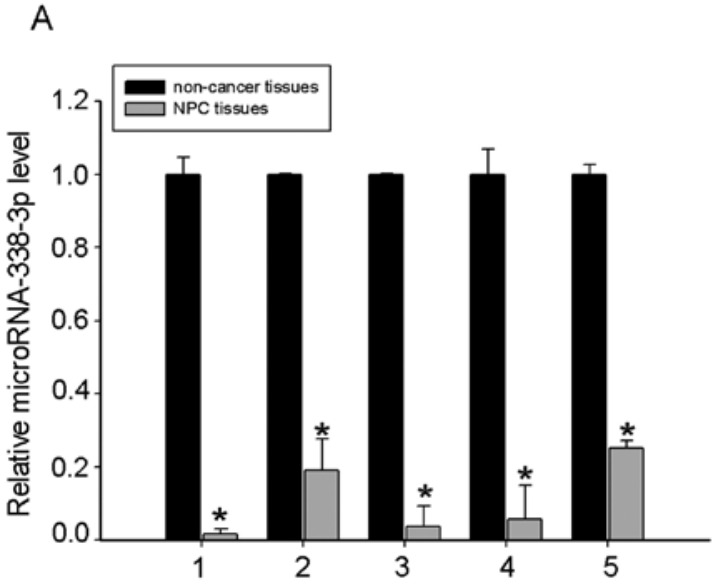

B

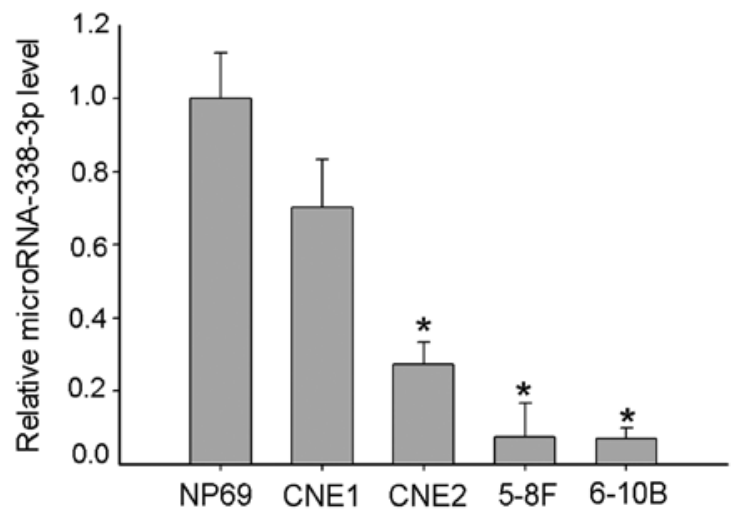

Figure 1. Expression of miR-338-3p in nasopharyngeal cancer (NPC) tissue samples and cells. (A) The expression levels of miR-338-3p in NPC and non-cancer tissues were measured by RT-qPCR. U6 snRNA was used as an internal control for miR-338-3p. Data are the means \pm SD. ${ }^{*} \mathrm{P}<0.05$. (B) miR-338-3p was decreased in the NPC cell lines (CNE1, CNE2, 6-10B and $5-8 \mathrm{~F}$ ) compared with the immortalized normal nasopharyngeal epithelial NP69 cells

\section{Results}

miR-338-3p is downregulated in NPC clinical specimens and cell lines. To determine whether miR-338-3p is involved in regulation of human NPC tumorigenesis, we firstly tested miR-338-3p expression in 5 freshly-frozen NPC and normal nasopharyngeal epithelial tissue samples and found that the miR-338-3p expression was significantly downregulated in NPC tissues (Fig. 1A). We also found miR-338-3p underexpressed in NPC cell lines CNE1, CNE2, 6-10B and 5-8F, compared to the normal nasopharyngeal epithelial cell line NP69 (Fig. 1B). Taken together, these findings suggested that miR-338-3p was downregulated in NPC.

miR-338-3p directly targets oncogenic HIF-l $\alpha$. miRNAs have been shown to target specific genes to regulate the progression of NPC. To identify miR-338-3p target genes, we used the target prediction program, Bioinformatic analysis using the TargetScan (http://www.targetscan.org) and miRanda algorithm (http://www.microrna.org) indicated that the 3'-UTR of HIF-1 $\alpha$ contained a predicted binding site for miR-338-3p (Fig. 2A). HIF-1 $\alpha$ increased in the NPC cells under hypoxic conditions. Compared with the other NPC cell lines CNE1, 

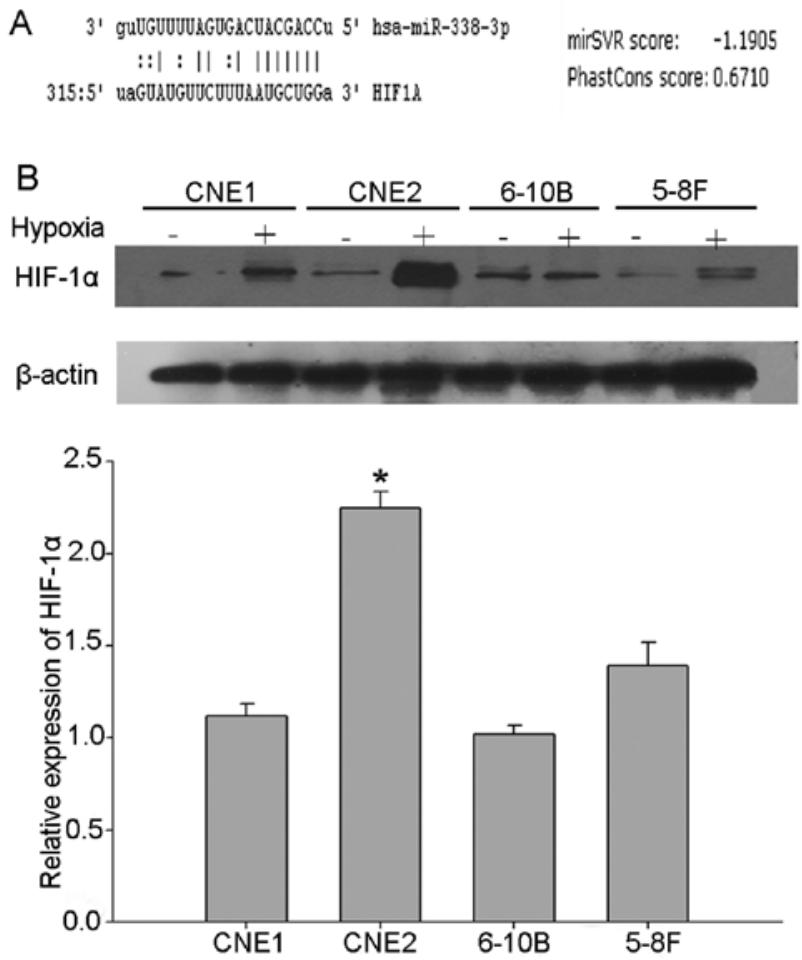

C

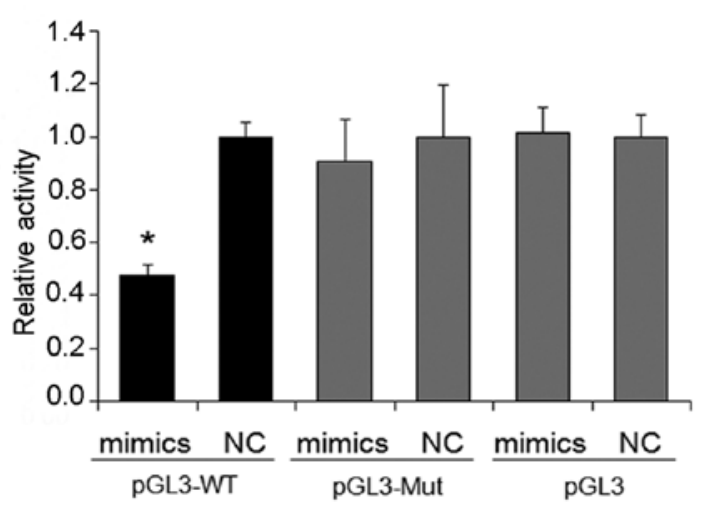

Figure 2. miR-338-3p directly targets oncogenic HIF-1 $\alpha$. (A) Predicted miR-338-3p target sequences in the HIF-1 $\alpha$ 3'-UTR. (B) Western blot analysis of HIF-1 $\alpha$ levels in NPC cells. $\beta$-actin was used as control. Cells were cultured under normal or hypoxic conditions at $24 \mathrm{~h}$. (C) Luciferase reporter assay of cells transfected with the wild-type (wt) or mutant (mut) HIF-1 $\alpha$ 3'-UTR luciferase reporter plasmid of negative control miRNA (NC) or miR-338-3p in NPC cells two days post-transfection.

6-10B and $5-8 \mathrm{~F}$, as shown by western blot analysis, HIF-1 $\alpha$ increased most in CNE2 cells (Fig. 2B). Then CNE2 cells were chosen for the following experiments. HIF-1 $\alpha$ has been reported to be an important molecule that drives cancer cell proliferation, migration and invasion including NPC. Using prediction tools, we predicted that the target of miR-338-3p is HIF-1 $\alpha$. To demonstrate that miR-338-3p directly targets HIF-1 $\alpha$ by interacting with its 3'-UTR, we co-transfected the pGL luciferase reporter plasmid harboring the wild-type or mutant 3'-UTR of HIF-1 $\alpha$, along with miR-338-3p or NC miRNA (Fig. 2C). Overexpressed miR-338-3p resulted in significant reduction of HIF-1 $\alpha$ 3'-UTR firefly luciferase reporter activity containing wild-type but not mutant binding sites compared to that of NC-miRNA. In summary, these results indicate that HIF-1 $\alpha$ was a direct target gene of miR-338-3p in NPC cells.
To examine the functional significance of miR-338-3p in NPC, we infected the CNE2 cells with miR-338-3p mimics and inhibitor, using miR-67 as control. Using RT-qPCR, we confirmed that the miR-338-3p level had increased $>20$-fold after transfection with miR-338-3p mimics compared with the expression of cont-miR, while the level was downregulated after the miR-338-3p inhibitor was delivered into CNE2 cells (Fig. 3A). To further investigate the antitumor effect of miR-338-3p under hypoxic conditions and to monitor the effect of miR-338-3p overexpression on HIF-1 $\alpha$ expression in target cells expressing low levels of miR-338-3p, NPC cells transfected with miR-338-3p mimics, inhibitors and scrambled control were exposed to hypoxic conditions for $24 \mathrm{~h}$. To confirm that miR-338-3p regulates HIF-1 $\alpha$ expression, we assessed HIF-1 $\alpha$ protein levels in CNE2 cells expressing ectopic miR-338-3p, using western blot analysis. The results showed that HIF-1 $\alpha$ levels, under hypoxia, were consistently downregulated by overexpressed miR-338-3p in CNE2 cell lines (Fig. 3B). These results were substantiated by immunofluorescence microscopy (Fig. 3C). Immunofluorescence staining revealed that HIF-1 $\alpha$ expression was inhibited by transfection with miR-338-3p mimics and was promoted by transfection with miR-338-3p inhibitor.

miR-338-3p regulates the metastasis potential of human NPC cell lines in vitro. To examine the functional significance of overexpressed miR-338-3p in NPC, we first investigated the metastasis potential of CNE2 cells with exceptional expression of miR-338-3p. Wound healing tests demonstrated that hypoxic condition increased the metastasis capacity of CNE2 cells. However, the overexpression of miR-338-3p significantly reduced the metastasis of the CNE2 cells under hypoxia (Fig. 4A), the results were confirmed by Transwell analysis (Fig. 4B).

To further explore the molecular mechanism of miR-338-3p on hypoxic signaling in NPC cells, the factors responsible for hypoxia were investigated after the treatment of hypoxic CNE2. Our previous study by western blot analysis disclosed that miR-338-3p downregulated the expression level of HIF-1 $\alpha$, showed that hypoxia selects for survival the more aggressive tumor cells and induces epithelial to mesenchymal transition (EMT) (29). We suspected that miRNAs would affect EMT under hypoxia since hypoxia is a key factor in the process of EMT. As shown here, exposured CNE2 cells to hypoxia resulted in the transition to a mesenchymal morphology, significant loss of E-cadherin and increased expression of vimentin. To assess the effect of miR-338-3p on hypoxiamediated EMT, miR-338-3p mimics, inhibitor and scrambled control was transfected into CNE2 cells, and the cells were moved to the hypoxic incubator $\left(1 \% \mathrm{O}_{2}, 5 \% \mathrm{CO}_{2}, 37^{\circ} \mathrm{C}\right)$ for another 24-h incubation. As shown in Fig. $4 \mathrm{C}$, the downstream E-cadherin was upregulated and vimentin was downregulated in miR-338-3p mimic-pretreated CNE2, respectively.

miR-338-3p suppresses NPC cell viability under hypoxic conditions. In order to investigate the effect of miR-338-3p on NPC cell proliferation, CCK8 assays were employed to analyze cell proliferation. As shown in Fig. 5A, overexpression of miR-338-3p in CNE2 cells significantly inhibited cell proliferation at first $24 \mathrm{~h}$ under hypoxic conditions $(\mathrm{P}<0.05)$, compared 


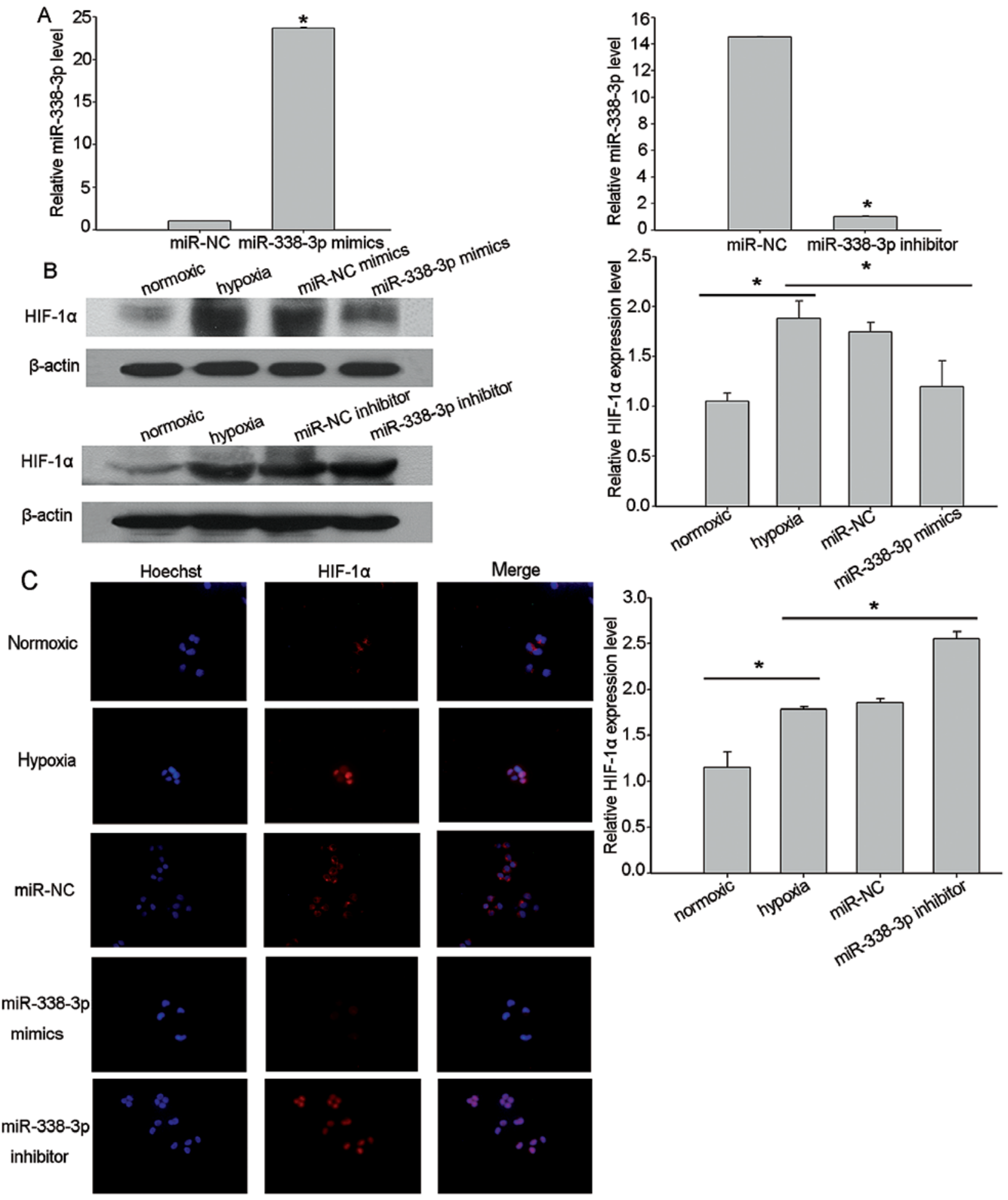

Figure 3. miR-338-3p regulates the expression of HIF-1 $\alpha$. (A) Relative miR-338-3p levels in miR-338-3p mimics and inhibitor transfected CNE2 cells. Transcript levels were normalized to U6 expression. (B) Western blot analysis of HIF-1 $\alpha$ levels in NC- or miR-338-3p-transfected cells. $\beta$-actin was used as loading control. Cells were cultured under hypoxia at two days post-transfection with cells cultured under normoxia as reference, and $24 \mathrm{~h}$ later protein levels were analyzed. (C) Immunofluorescence staining analysis of cytoplasmic and nuclear expression of HIF-1 $\alpha$ in NC- or miR-338-3p-transfected CNE2 cells. Cells were incubated under hypoxia for $24 \mathrm{~h}$. Red is HIF-1 $\alpha$ staining. Blue is the nuclear staining by Hochest. Data are shown as mean \pm SEM of three independent experiments. ${ }^{*} \mathrm{P}<0.05$.

with the NC transfection. To further demonstrate the effect of miR-338-3p on cell proliferation, miR-338-3p inhibitor was transfected into CNE2 cells. Compared with the mock group, the proliferation of the miR-338-3p inhibitor transfected cells significantly increased (Fig. 5B). Previous evidence showed that miR-338-3p regulated the phosphorylation of ERK1/2 to mediate tumor cell metastasis and proliferation in gastric cancer (22). Thus, we assumed that miR-338-3p could decrease the expression of the phosphorylation of Erk1/2 by targeting HIF-1 $\alpha$ under hypoxic conditions. Our data showed that the 
A

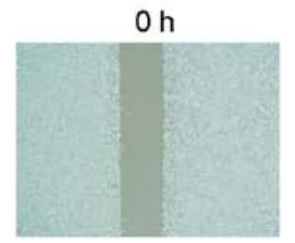

miR-67 mimics

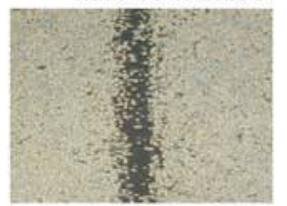

miR-67 inhibitor

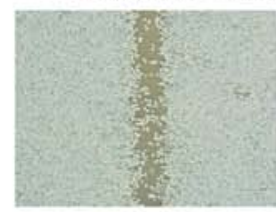

B

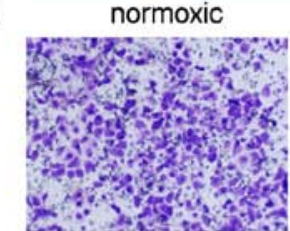

miR-NC mimics

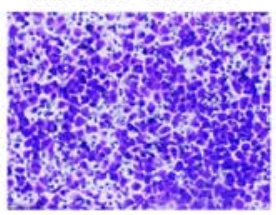

miR-NC inhibitor

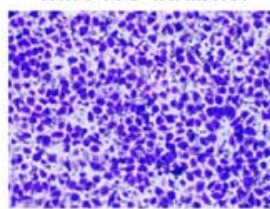

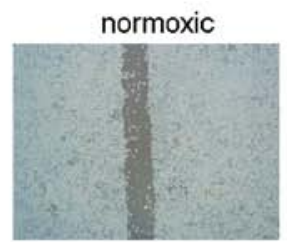

miR-338-3p mimics

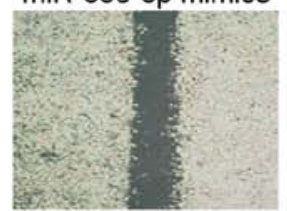

miR-338-3p inhibitor

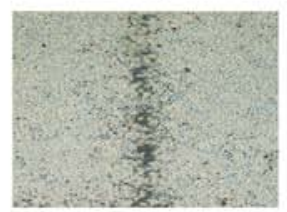

hypoxia

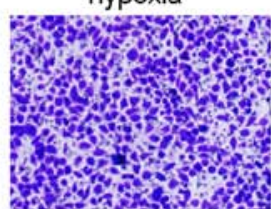

miR-338-3p mimics

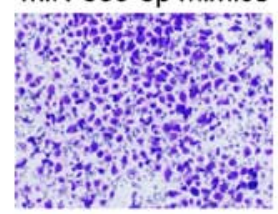

miR-338-3p inhibitor

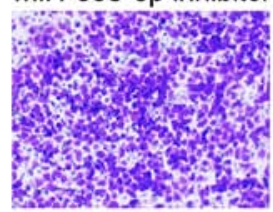

hypoxia

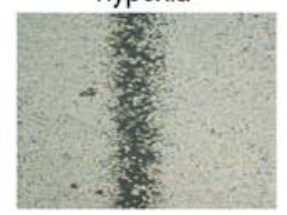

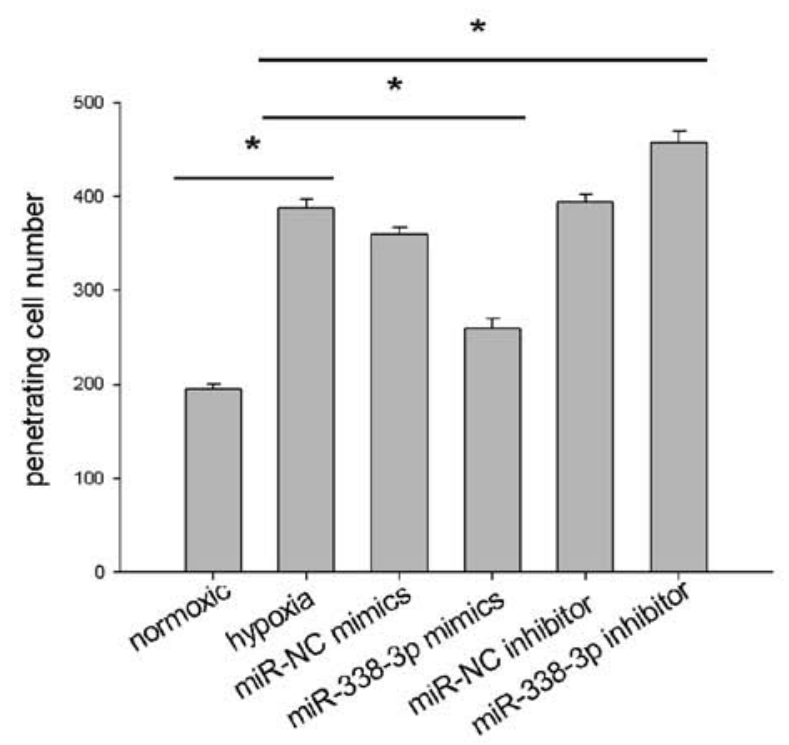
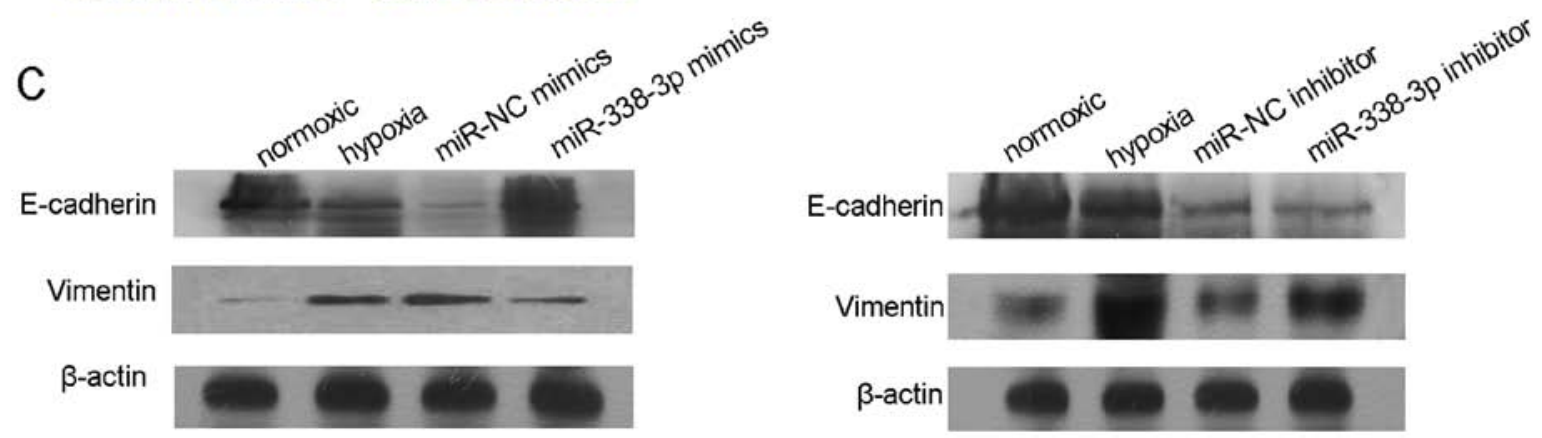

Figure 4. miR-338-3p regulates the metastasis potential of human NPC cell lines in vitro. (A) Cells transfected with miR-338-3p mimics showed a slower wound closure rate than the cells transfected with the non-specific control or miR-338-3p inhibitor under hypoxic conditions. (B) Transwell analysis confirmed the results above that the overexpression of miR-338-3p significantly reduced the metastasis capacity of the CNE2 cells under hypoxic conditions. Data are presented as mean number of migrated cells per HPF (five random fields were analyzed), compared with the control. ${ }^{*} \mathrm{P}<0.05$. (C) Western blot analysis of E-cadherin, and vimentin expression in parental cells, miR-338-3p and miRNA control transfected cells after $24 \mathrm{~h}$ under hypoxic conditions compared with cells cultured under normal conditions. A representative blot from three independent experiments is shown. $\beta$-actin was used as control.

overexpression of miR-338-3p inhibited the phosphorylation of Erk1/2, but the relative expression level of total Erk1/2 was not significantly altered (Fig. 5C). Taken together, these results suggested anti-cell growth properties of miR-338-3p in CNE2 cells.
miR-338-3p sensitizes NPC cells to cisplatin. Because recent studies have reported that destabilization of HIF-1 $\alpha$ can overcome hypoxia-mediated cisplatin resistance in nonsmall cell lung carcinoma (30), we then tested whether miR-338-3p could sensitize NPC cells to chemotherapy 
A
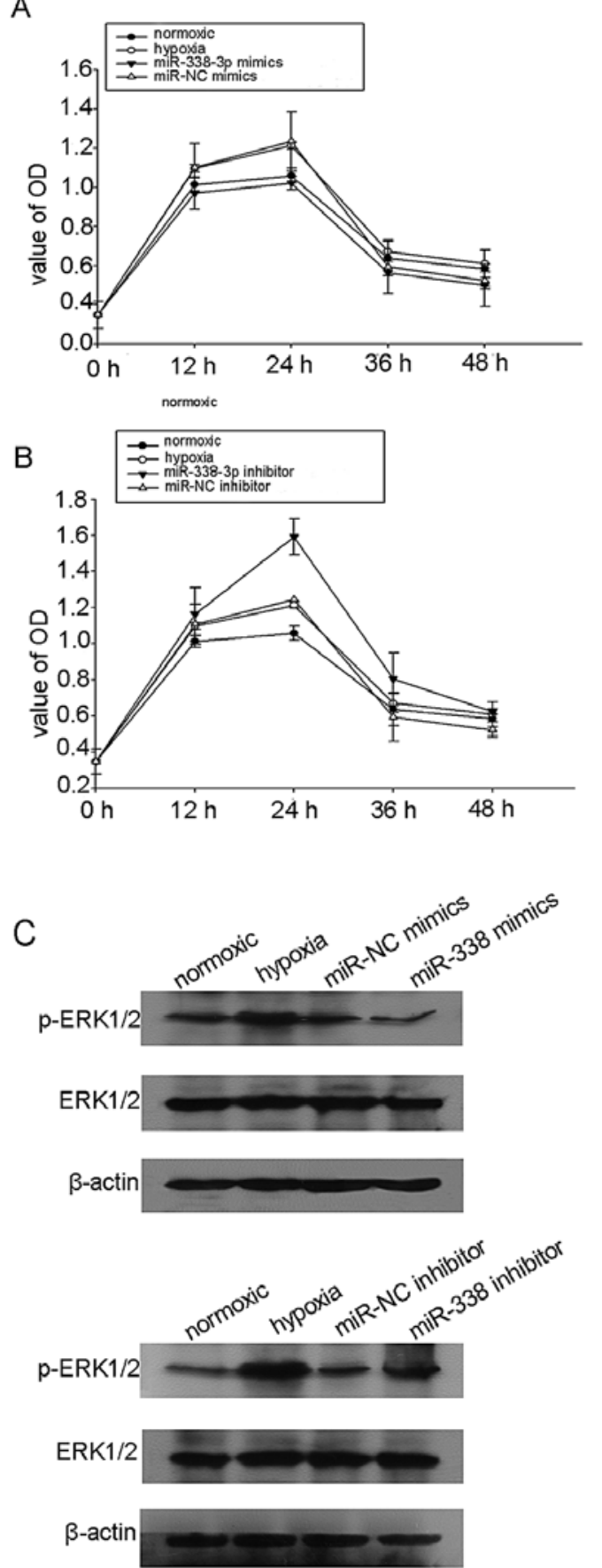

Figure 5. miR-338-3p inhibited CNE2 cell proliferation in vitro. (A) The CCK8 assay showed that miR-338-3p inhibited CNE2 cells viability. The CCK8 assay was performed after transfection of miR-338-3p mimics and miR-NC into the CNE2 cells. (B) The cell viability of CNE2 cells treated with miR-338-3p inhibitor compared to normaxic, hypoxia or miR-NC transfected cells. (C) Western blot analysis showed downregulation of p-ERK induced by miR-338-3p in hypoxic CNE2 cells. $\beta$-actin was used as an internal control.

treatment of cisplatin. CCK 8 assay showed that 5 and $10 \mu \mathrm{g} / 1$ cisplatin inhibited the proliferation of CNE-2 cells in a dose- and time-dependent manner (Fig. 6A). However, the inhibition effect of $2.5 \mu \mathrm{g} / \mathrm{l}$ cisplatin on hypoxic CNE2 cells was not obvious. To determine the chemo-sensitizing effects of miR-338-3p on hypoxic NPC cells, we chose the treatment dose at $2.5 \mu \mathrm{g} / 1$ for the following experiments. We treated miR-338-3p transfected cells and NC cells with
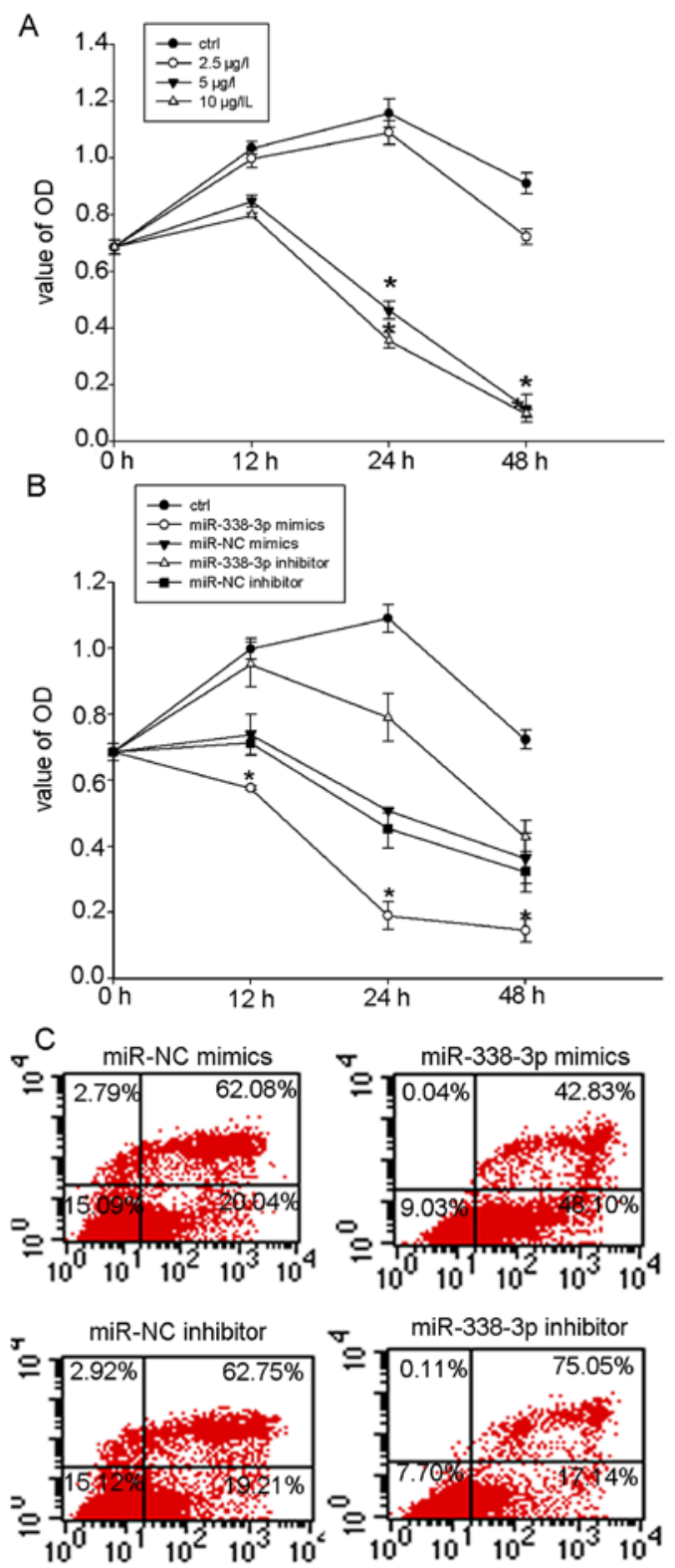

Figure 6. miR-338-3p sensitizes NPC cells to cisplatin. (A) Cell viability was determined in CNE2 cells after cisplatin $(0,2.5,5$ and $10 \mathrm{mM})$ treatment under hypoxia. (B) The CCK8 assay showed that the CNE2 cells transfected with miR-338-3p mimics were significantly more sensitive to cisplatin therapy of $2.5 \mu \mathrm{g} / 1$ than control cells under hypoxic condition ( $\mathrm{P}<0.05)$. Data are mean \pm SD of three experiments. (C) Annexin V/PI double staining showing percentage of early and late apoptotic cells in miR-NC or miR-338-3p transfected CNE2 cells under hypoxic conditions.

cisplatin and measured the cell viability. Cell viability (CCK8 assay) showed that non-transfected CNE2 cells were highly resistant to cisplatin $(2.5 \mu \mathrm{g} / \mathrm{l})$ under hypoxia and that transfection with miR-338-3p mimics significantly reduced cisplatin resistance (Fig. 6B). To determine whether the chemosensitizing effects of miR-338-3p on NPC cells result from the induction of apoptosis, we treated miR-338-3p transfected CNE-2 cells in hypoxic conditions for $24 \mathrm{~h}$. As shown in Fig. 6C, the rate of apoptotic cell death was significant higher in cells treated with miR-338-3p mimics (Fig. 6C). The above data supported the indicated potential applications for miR-338-3p in anticancer therapy. 


\section{Discussion}

The present study focused on the antitumor effect of miR-338-3p in NPC. We demonstrated that the overexpressed miR-338-3p was able to inhibit the proliferation and migration of hypoxic CNE2 cells as well as overcome hypoxia-mediated cisplatin resistance.

NPC is a highly invasive malignancy, and up to $70 \%$ of patients with NPC present with a locally advanced stage or with cervical lymph node metastasis at the time of diagnosis $(2,25)$. Those patients have a poor outcome, and drug resistance may hamper the efficacy of anticancer drugs (2). Therefore, clarification of the molecular pathogenesis and mechanism of NPC is crucial for developing effective therapy strategies to improve the outcome of patients with this disease.

Growing evidence indicates that miRNAs hold great promise for novel therapeutic approaches for treating human cancers (28) and that hypoxia regulated miRNAs exhibit induction in response to HIF activation and participate in the development of angiogenesis and tumorigenesis (31). Previous studies have demonstrated that miR-15b, miR-16, miR-20a, and miR-20b are sharply downregulated in CNE cells during hypoxia (31).

Many studies have reported on the tumor suppressive effects of miR-338-3p in the malignant processes of various cancers, such as gastric cancer (19) hepatocellular carcinoma (20) and malignant melanoma (21). However, there is little knowledge on miR-338-3p and its targets in NPC. Our data showed that miR-338-3p expression is markedly downregulated in NPC patient samples and NPC cell lines as compared to immortalized nasopharyngeal epithelial cells.

Predicted targets of miR-338-3p are elements involved in many biological processes, such as cell proliferation, differentiation, metastasis and cell death in various types of cancer $(28,32)$. Similar to previous studies, our data identified HIF-1 $\alpha$ as a key target of miR-338-3p. HIF-1 $\alpha$ is an important hypoxia transcription molecule promoting angiogenesis and metastasis.

Hypoxia is one of the fundamental biological properties that are associated with the development and aggressiveness of a variety of solid tumors $(33,34)$, including NPC (24). Previous study shows that all primary NPC tumors contain hypoxic regions which result in decreased local control and increased distant metastases, as well as resistance to chemotherapy in NPC patients (25). It was recently reported that hypoxia inducible factor (HIF) results in global transcriptional changes in gene expression, playing an important role in promoting tumor progression, angiogenesis and metastasis $(34,35)$. In the present study, we showed evidence that the HIF-1 $\alpha$ levels were consistently downregulated by overexpressed miR-338-3p in CNE2 cell lines under hypoxia conditons. Previous studies have shown that the oxygen-sensitive HIF-1 $\alpha$ subunit accumulates and translocates into the nucleus under hypoxia conditions, where it binds to the constitutively expressed HIF-1 $\beta$, forming the active HIF-1 heterodimer (36). In the present study, immunofluorescence staining results revealed that HIF-1 $\alpha$ was accumulated into the nuclei in hypoxia cells. However, the overall staining and nuclear accumulation of HIF-1 $\alpha$ was reduced after miR-338-3p transfection. Furthermore, overexpressed miR-338-3p inhibited cell viability as well as metastasis ability by directly targeting HIF-1 $\alpha$.
HIF-1 $\alpha$ activates some important oxygen modulated genes critically involved in tumor angiogenesis and metastasis (37). EMT is clearly associated with pathological processes requiring epithelial cell migration and invasion $(2,38)$. Furthermore, HIF-1 $\alpha$ also initiates endothelial to mesenchymal transition (39). Our data showed that the over-expressed miR-338-3p inhibited the hypoxia-induced EMT.

HIF-1 $\alpha$ has attracted considerable interest as a potential target in cancer therapy (37). Notably, growing evidence suggests that higher HIF-1 $\alpha$ levels in tumors are related to radioresistance and poor clinical outcome in different tumor entities (40). Some evidence shows that HIF-1 $\alpha$-deficient cells are more sensitive to radiotherapy and chemotherapy compared with normal cells (41), however, the mechanism needs further investigation. Therefore, the specific inhibition of HIF may enhance cancer radiosensitivity in clinical settings. Our results showed that miR-338-3p downregulated the level of HIF-1 $\alpha$ in CNE2 under hypoxic conditions and could overcome hypoxia-mediated cisplatin resistance.

Previous studies have shown that other cell regulatory elements such as cyclin D, and smoothened, are also targets of miR-338-3p that are aberrantly expressed due to the downregulation of miR-338-3p in HCC (28). Undoubtedly, regulation of these other targets may contribute to the inhibitory effects of miR-338-3p on NPC. However, considering our observation that HIF-1 $\alpha$ overexpression rescued the cell from the anti-NPC activity of miR-338-3p, it is likely that regulation of HIF-1 $\alpha$ by miR-338-3p is a key antitumor aspect in NPC. Our further studies will focus on other targets of miR-338-3p and their specific roles under both normoxic and hypoxic conditions.

In conventional clinical therapy of NPC, radiotherapy is the primary therapeutic approach, while using radiotherapy combined with chemotherapy is recommended for the treatment of advanced carcinoma (42). Treatment failure rates remain high and the 5-year survival rate is extremely low (42). One reason for the treatment failure may be attributed to the drug resistance and distant metastasis (42). Evidence shows that destabilization of HIF-1 $\alpha$ can overcome hypoxia-mediated cisplatin resistance in non-small cell lung carcinoma (30). We want to determine whether miR-338-3p potentiates sensitivity of NPC cells to cisplatin, which is clinically used as adjuvant therapy for NPC in order to induce tumor cell death (42). Our results showed that under hypoxic conditions, CNE2 cells are highly resistant to cisplatin in vitro. Moreover, cells pretransfected with miR-338-3p can overcome hypoxia-mediated cisplatin resistance. These results enriched the function of miR-338-3p in addition to its role as a tumor suppressor.

In conclusion, we found that miR-338-3p was downregulated in NPC clinical samples and cell lines. HIF-1 $\alpha$ was verified as a direct target of miR-338-3p, and involved in NPC cell growth and invasion. Furthermore, our data suggest that miR-338-3p and/ or its target gene HIF-1 $\alpha$ could represent important therapeutic targets in NPC. We explored its effects on cell growth, invasive and chemotherapy resistance. The miR-338-3p/HIF-1 $\alpha$ pathway may help to understand the mechanisms of NPC progression and would provide a novel therapeutic strategy for NPC.

\section{Acknowledgements}

The present study was supported by grants from the Chinese National Natural Science Foundation (nos. 81172841, 81202368 
and 81471603), the China Postdoctoral Science Foundation (2013M541708); the project of '333 Natural Science Foundation' of Jiangsu Grant (BRA2013286); the 'Top Six Types of Talents' Financial Assistance of Jiangsu Province Grant (no. 6); the project of Jiangsu Provincial Health Department (Z201005); the innovative project o Nantong University postgraduate students (13025043); the Jiangsu province's Outstanding Medical Academic Leader Program (LJ201136); and the Natural Science Foundation of Jiangsu Province (SBK2015022581).

\section{References}

1. Liu N, Jiang N, Guo R, Jiang W, He QM, Xu YF, Li YQ, Tang LL, Mao YP, Sun Y, et al: MiR-451 inhibits cell growth and invasion by targeting MIF and is associated with survival in nasopharyngeal carcinoma. Mol Cancer 12: 123, 2013.

2. Aga M, Bentz GL, Raffa S, Torrisi MR, Kondo S, Wakisaka N, Yoshizaki T, Pagano JS and Shackelford J: Exosomal HIF1a supports invasive potential of nasopharyngeal carcinomaassociated LMP1-positive exosomes. Oncogene 33: 4613-4622, 2014.

3. Krutilina R, Sun W, Sethuraman A, Brown M, Seagroves TN, Pfeffer LM, Ignatova T and Fan M: MicroRNA-18a inhibits hypoxia-inducible factor $1 \alpha$ activity and lung metastasis in basal breast cancers. Breast Cancer Res 16: R78, 2014.

4. Long Z, Wang B, Tao D, Huang Y and Tao Z: Hypofractionated radiotherapy induces miR-34a expression and enhances apoptosis in human nasopharyngeal carcinoma cells. Int J Mol Med 34 1388-1394, 2014

5. Zhou W, Fong MY, Min Y, Somlo G, Liu L, Palomares MR, Yu Y, Chow A, O'Connor ST, Chin AR, et al: Cancer-secreted miR-105 destroys vascular endothelial barriers to promote metastasis. Cancer Cell 25: 501-515, 2014.

6. Suh YE, Raulf N, Gäken J, Lawler K, Urbano TG, Bullenkamp J, Gobeil S, Huot J, Odell E and Tavassoli M: MicroRNA-196a promotes an oncogenic effect in head and neck cancer cells by suppressing annexin A1 and enhancing radioresistance. Int J Cancer 137: 1021-1034, 2014

7. Huang XH, Chen JS, Wang Q, Chen XL, Wen L, Chen LZ, Bi J, Zhang LJ, Su Q and Zeng WT: miR-338-3p suppresses invasion of liver cancer cell by targeting smoothened. J Pathol 225: 463-472, 2011

8. Lu ZJ, Lu LG, Tao KZ, Chen DF, Xia Q, Weng JJ, Zhu F, Wang XP and Zheng P: MicroRNA-185 suppresses growth and invasion of colon cancer cells through inhibition of the hypoxia-inducible factor- $2 \alpha$ pathway in vitro and in vivo. Mol Med Rep 10: 2401-2408, 2014

9. Luo Z, Dai Y, Zhang L, Jiang C, Li Z, Yang J, McCarthy JB, She $\mathrm{X}$, Zhang $\mathrm{W}$, Ma J, et al: miR-18a promotes malignant progression by impairing microRNA biogenesis in nasopharyngeal carcinoma. Carcinogenesis 34: 415-425, 2013.

10. Liu N, Tang LL, Sun Y, Cui RX, Wang HY, Huang BJ, He QM, Jiang W and Ma J: MiR-29c suppresses invasion and metastasis by targeting TIAM1 in nasopharyngeal carcinoma. Cancer Lett 329: 181-188, 2013.

11. Zhang JX, Qian D, Wang FW, Liao DZ, Wei JH, Tong ZT, Fu J, Huang XX, Liao YJ, Deng HX, et al: MicroRNA-29c enhances the sensitivities of human nasopharyngeal carcinoma to cisplatinbased chemotherapy and radiotherapy. Cancer Lett 329: 91-98, 2013.

12. Alajez NM, Lenarduzzi M, Ito E, Hui AB, Shi W, Bruce J, Yue S, Huang $\mathrm{SH}, \mathrm{Xu}$ W, Waldron J, et al: MiR-218 suppresses nasopharyngeal cancer progression through downregulation of survivin and the SLIT2-ROBO1 pathway. Cancer Res 71: 2381-2391, 2011.

13. Lu J, He ML, Wang L, Chen Y, Liu X, Dong Q, Chen YC, Peng Y, Yao KT, Kung HF, et al: MiR-26a inhibits cell growth and tumorigenesis of nasopharyngeal carcinoma through repression of EZH2. Cancer Res 71: 225-233, 2011.

14. Ji Y, He Y, Liu L and Chong X: MiRNA-26b regulates the expression of cyclooxygenase-2 in desferrioxamine-treated CNE cells. FEBS Lett 584: 961-967, 2010.

15. Deng M, Tang H, Zhou Y, Zhou M, Xiong W, Zheng Y, Ye Q, Zeng X, Liao Q, Guo X, et al: miR-216b suppresses tumor growth and invasion by targeting KRAS in nasopharyngeal carcinoma. J Cell Sci 124: 2997-3005, 2011.
16. Li G, Wu Z, Peng Y, Liu X, Lu J, Wang L, Pan Q, He ML and Li XP: MicroRNA-10b induced by Epstein-Barr virus-encoded latent membrane protein-1 promotes the metastasis of human nasopharyngeal carcinoma cells. Cancer Lett 299: 29-36, 2010.

17. Zhang L, Deng T, Li X, Liu H, Zhou H, Ma J, Wu M, Zhou M, Shen S, Li X, et al: microRNA-141 is involved in a nasopharyngeal carcinoma-related genes network. Carcinogenesis 31 : 559-566, 2010.

18. Xia H, Ng SS, Jiang S, Cheung WK, Sze J, Bian XW, Kung HF and Lin MC: miR-200a-mediated downregulation of ZEB2 and CTNNB1 differentially inhibits nasopharyngeal carcinoma cell growth, migration and invasion. Biochem Biophys Res Commun 391: 535-541, 2010

19. Guo B, Liu L, Yao J, Ma R, Chang D, Li Z, Song T and Huang C: miR-338-3p suppresses gastric cancer progression through a PTEN-AKT axis by targeting P-REX2a. Mol Cancer Res 12: 313-321, 2014.

20. Huang XH, Wang Q, Chen JS, Fu XH, Chen XL, Chen LZ, Li W Bi J, Zhang LJ, Fu Q, et al: Bead-based microarray analysis of microRNA expression in hepatocellular carcinoma: miR-338 is downregulated. Hepatol Res 39: 786-794, 2009.

21. Caramuta S, Egyházi S, Rodolfo M, Witten D, Hansson J, Larsson C and Lui WO: MicroRNA expression profiles associated with mutational status and survival in malignant melanoma. J Invest Dermatol 130: 2062-2070, 2010.

22. Peng Y, Liu YM, Li LC, Wang LL and Wu XL: MicroRNA-338 inhibits growth, invasion and metastasis of gastric cancer by targeting NRP1 expression. PLoS One 9: e94422, 2014.

23. Kucharzewska P, Christianson HC, Welch JE, Svensson KJ, Fredlund E, Ringnér M, Mörgelin M, Bourseau-Guilmain E, Bengzon $\mathrm{J}$ and Belting M: Exosomes reflect the hypoxic status of glioma cells and mediate hypoxia-dependent activation of vascular cells during tumor development. Proc Natl Acad Sci USA 110: 7312-7317, 2013.

24. Chen Y, Li X, Wu S, Xu G, Zhou Y, Gong L, Li Z and Yang D: Expression of HIF-1 $\alpha$ and CAIX in nasopharyngeal carcinoma and their correlation with patients' prognosis. Med Oncol 31: 304 2014.

25. Pan WL, Wong JH, Fang EF, Chan YS, Ng TB and Cheung RC: Preferential cytotoxicity of the type I ribosome inactivating protein alpha-momorcharin on human nasopharyngeal carcinoma cells under normoxia and hypoxia. Biochem Pharmacol 89: 329-339, 2014.

26. Xueguan L, Xiaoshen W, Yongsheng Z, Chaosu H, Chunying S and Yan F: Hypoxia inducible factor-1 alpha and vascular endothelial growth factor expression are associated with a poor prognosis in patients with nasopharyngeal carcinoma receiving radiotherapy with carbogen and nicotinamide. Clin Oncol ( R Coll Radiol) 20: 606-612, 2008.

27. Hui EP, Chan AT, Pezzella F, Turley H, To KF, Poon TC, Zee B, Mo F, Teo PM, Huang DP, et al: Coexpression of hypoxiainducible factors 1alpha and 2alpha, carbonic anhydrase IX, and vascular endothelial growth factor in nasopharyngeal carcinoma and relationship to survival. Clin Cancer Res 8: 2595-2604, 2002.

28. Xu H, Zhao L, Fang Q, Sun J, Zhang S, Zhan C, Liu S and Zhang Y: MiR-338-3p inhibits hepatocarcinoma cells and sensitizes these cells to sorafenib by targeting hypoxia-induced factor 1 $\alpha$. PLoS One 9: e115565, 2014.

29. Mak P, Leav I, Pursell B, Bae D, Yang X, Taglienti CA, Gouvin LM, Sharma VM and Mercurio AM: ERbeta impedes prostate cancer EMT by destabilizing HIF-lalpha and inhibiting VEGF-mediated snail nuclear localization: Implications for Gleason grading. Cancer Cell 17: 319-332, 2010.

30. Fischer C, Leithner K, Wohlkoenig C, Quehenberger F, Bertsch A, Olschewski A, Olschewski $\mathrm{H}$ and Hrzenjak A: Panobinostat reduces hypoxia-induced cisplatin resistance of non-small cell lung carcinoma cells via HIF-1 $\alpha$ destabilization. Mol Cancer 14: $4,2015$.

31. Du R, Sun W, Xia L, Zhao A, Yu Y, Zhao L, Wang H, Huang C and Sun S: Hypoxia-induced down-regulation of microRNA-34a promotes EMT by targeting the Notch signaling pathway in tubular epithelial cells. PLoS One 7: e30771, 2012.

32. Sun K, Deng HJ, Lei ST, Dong JQ and Li GX: miRNA-338-3p suppresses cell growth of human colorectal carcinoma by targeting smoothened. World J Gastroenterol 19: 2197-2207, 2013.

33. Bao B, Azmi AS, Ali S, Ahmad A, Li Y, Banerjee S, Kong D and Sarkar FH: The biological kinship of hypoxia with CSC and EMT and their relationship with deregulated expression of miRNAs and tumor aggressiveness. Biochim Biophys Acta 1826: 272-296, 2012. 
34. King HW, Michael MZ and Gleadle JM: Hypoxic enhancement of exosome release by breast cancer cells. BMC Cancer 12: 421, 2012.

35. Ruan K, Song G and Ouyang G: Role of hypoxia in the hallmarks of human cancer. J Cell Biochem 107: 1053-1062, 2009.

36. Zhou J, Li K, Gu Y, Feng B, Ren G, Zhang L, Wang Y, Nie Y and Fan D: Transcriptional up-regulation of RhoE by hypoxiainducible factor (HIF)-1 promotes epithelial to mesenchymal transition of gastric cancer cells during hypoxia. Biochem Biophys Res Commun 415: 348-354, 2011.

37. Luo Z, Bai M, Xiao X, Zhang W, Liu X, Yang X, Li S, Huan Y, Wu Z, Zhang X, et al: Silencing of HIF-1 $\alpha$ enhances the radiation sensitivity of human glioma growth in vitro and in vivo. Neuropharmacology 89: 168-174, 2015.

38. Li X, Zhang Z, Beiter T and Schluesener HJ: Nanovesicular vaccines: Exosomes. Arch Immunol Ther Exp (Warsz) 53 329-335, 2005.
39. Zhang L, Huang G, Li X, Zhang Y, Jiang Y, Shen J, Liu J, Wang Q, Zhu J, Feng X, et al: Hypoxia induces epithelial-mesenchymal transition via activation of SNAI1 by hypoxia-inducible factor $-1 \alpha$ in hepatocellular carcinoma. BMC Cancer 13: 108, 2013.

40. Meijer TW, Kaanders JH, Span PN and Bussink J: Targeting hypoxia, HIF-1, and tumor glucose metabolism to improve radiotherapy efficacy. Clin Cancer Res 18: 5585-5594, 2012.

41. Sasabe E, Zhou X, Li D, Oku N, Yamamoto T and Osaki T: The involvement of hypoxia-inducible factor-1alpha in the susceptibility to gamma-rays and chemotherapeutic drugs of oral squamous cell carcinoma cells. Int J Cancer 120: 268-277, 2007.

42. Zhang P, Liu H, Xia F, Zhang QW, Zhang YY, Zhao Q, Chao ZH, Jiang ZW and Jiang CC: Epithelial-mesenchymal transition is necessary for acquired resistance to cisplatin and increases the metastatic potential of nasopharyngeal carcinoma cells. Int J Mol Med 33: 151-159, 2014. 